\title{
ON ALGEBRAIC DIFFERENTIAL EQUATIONS SATISFIED BY SOME ELLIPTIC FUNCTIONS (II)
}

\author{
By \\ P. CHOWLA and S. CHOWLA
}

\section{Summary :}

In (I) we obtained the "implicit" algebraic differential equation for the function defined by

$$
Y=\sum_{1}^{\infty} \frac{n^{a} x^{n}}{1-x^{n}}
$$

where $a$ is an odd positive integer, and conjectured that there are no algebraic differential equations for the case when $a$ is an even integer. In this note we obtain a simple proof that (this has been known for almost 200 years )

$$
Y=\sum_{1}^{\infty} x^{n^{2}} \quad(|x|<1)
$$

satisfies an algebraic differential equation, and conjecture that

$$
\mathrm{Y}=\sum_{1}^{\infty} \mathrm{x}^{\mathrm{n}^{\mathrm{k}}}
$$

does not satisfy an algebraic differential equation.

$\$ 1$ In the standard notation of elliptic functions we write

$$
\mathrm{K}=\int_{0}^{\pi / 2} \frac{\mathrm{d} \phi}{\sqrt{-\mathrm{k}^{2} \sin ^{2} \phi}}
$$




$$
E=\int_{0}^{\pi / 2} \sqrt{1-k^{2} \sin ^{2} \phi} d \phi
$$

Here $0<\mathrm{k}<1$. We also define $\mathrm{k}^{\prime}$ by

$$
\mathrm{k}^{2}+\mathrm{k}^{2}=1
$$

and the corresponding Integrals

$$
\begin{aligned}
& K^{\prime}=\int_{0}^{\pi / 2} \frac{d \phi}{\sqrt{1-k^{\prime 2} \sin ^{2} \phi}} \\
& E^{\prime}=\int_{0}^{\pi / 2} \sqrt{1-k^{\prime 2} \sin ^{2} \phi} d \phi
\end{aligned}
$$

We know that

$$
\mathrm{KE}^{\prime}+\mathrm{K}^{\prime} \mathrm{E}-\mathrm{KK} \mathrm{K}^{\prime}=\frac{\pi}{2}
$$

which is often called Legendre's relation.

Our proof rests on several identities for $K, K^{\prime}, E, E^{\prime}$ and their derivatives. Most important :

$$
\sqrt{\frac{2 \boldsymbol{k}}{\pi}}=\sum_{-\infty}^{\infty} q^{n^{2}}
$$

where

$$
q=e^{-\frac{\pi K^{\prime}}{k}}
$$

§2. Next we need:

$$
\begin{aligned}
& \frac{\mathrm{dK}}{\mathrm{dk}}=\frac{E-\mathrm{k}^{{ }^{2}} \mathrm{~K}}{\mathrm{kk^{ \prime }}{ }^{2}}, \\
& \frac{\mathrm{dE}}{\mathrm{dk}}=\frac{\mathrm{E}-\mathrm{K}}{\mathrm{k}}
\end{aligned}
$$

Since

$$
\frac{\mathrm{d} \mathrm{k}^{\prime}}{\mathrm{d} \mathrm{k}}=-\frac{\mathrm{k}}{\mathrm{k}^{\prime}}
$$


we easily express

$$
\frac{d E^{\prime}}{d k}, \frac{d K^{\prime}}{d k}
$$

as easy functions of $K, K^{\prime}, E, E^{\prime}$ and $k$. From these

$$
\frac{d}{d k}\left(E K^{\prime}+K E^{\prime}-K K^{\prime}\right)=0
$$

Hence

$$
E K^{\prime}+K E^{\prime}-K K^{\prime}=c
$$

where $c$ is a constant independent of $k$.

We casily see that $c=\frac{\pi}{2}$.

§3. Next

$$
\frac{d q}{d k}=\frac{\pi^{2} q}{2 k k^{\prime 2} K^{2}}
$$

(Ramanujan, Collected Papers, p. 32)

f 4. Next find

$$
\frac{d K}{d q}=\frac{d K}{d k} \frac{d k}{d q}
$$

which is, from our previous relations a simple rational function of $\mathrm{K}, \mathrm{E}, \mathrm{k}, \mathrm{q}$.

Form

$$
\frac{d^{2} K}{d q^{2}} \text { and } \frac{d^{3} K}{d q^{3}}
$$

which again are simple rational functions of $K, E, k, q$. Eliminate $E$ and $k$ from the relations obtained in this section. We then get a "polynomia!" relation hetween 


$$
K, q, \frac{d K}{d q}, \frac{d^{2} K}{d q^{2}}, \frac{d^{3} K}{d q^{3}}
$$

which is a third order algebraic differential equation

$$
T\left(K, q, \frac{d K}{d q}, \frac{d^{2} K}{d^{2} q^{2}}, \frac{d^{3} K}{d q^{3}}\right)=0
$$

where $\mathbf{T}$ is a polynomial in the five quantities following $\mathbf{T}$.

15. It would be nice to obtain "explicitly" the polynomial T of the last section, as well as those mentioned in the beginning of this paper i.e. algebraic differential equations satisfied by

$$
Y=\sum_{1}^{\infty} \frac{n^{a} x^{n}}{1-x^{n}}
$$

i. e. a relation with an explicit polynomial $\mathrm{T}$ with

$$
\mathrm{T}\left(\mathrm{Y}, \mathrm{X}, \mathrm{Y}^{\prime}, \mathrm{Y}^{\prime \prime}, \mathrm{Y}^{\prime \prime \prime}\right)=0
$$

As far we know this has never been do ne.

\section{Conclusion.}

Professor Dwork (Princeton University) pointed out to us that G. Valerion in a monograph (in French) on automorphic forms in analysis obtained the algebraic differential equation explicitly in the case of the function

$$
Y=\underset{1}{\pi}\left(1-x^{n}\right) .
$$

